\title{
A Novel Approach to Optimize Energy in WSN using Bellman- Ford Asynchronous - SW Protocol
}

\author{
Ambrish M Salakki, Associate Prof. M A Mubeen \\ Department of E\&C, Shadan College of Engineering \&Technology, JNTU, Hyderabad,
}

\begin{abstract}
Energy optimization is a prime issue in wireless sensor network that consumes less energy to give optimum performance under low battery power, limited bandwidth and network life time of each node. In this paper, first we investigate and analyze three network layer protocols, centralized, localized, bellman ford(distributive) and next we develop a new algorithm called bellman ford asynchronous sleep wake up protocol for energy saving in packet transmission and to prolong the network lifetime. The simulation results on metrics energy spent, throughput, average delay and product delivery ratio confirms that the new protocol outperforms the other protocols.
\end{abstract}

Keywords: Localized, Centralized, Distributed, Asynchronous Sleep Wake Up.

\section{Introduction}

Recent advances in wireless communications, and digital electronics have enabled the development of low-cost, low-power, multifunctional sensor nodes that are small in size and communicate untethered in short distances. These tiny sensor nodes, which consist of sensing, data processing, and communicating components, leverage the idea of sensor networks based on collaborative effort of a large number of nodes. Sensor networks represent a significant improvement over traditional sensors, which are deployed in the following two ways

- Sensors can be positioned far from the actual phenomenon, i.e., something known by sense perception. In this approach, large sensors that use some complex techniques to distinguish the targets from environmental noise are required.

- Several sensors that perform only sensing can be deployed. The positions of the sensors and communications topology are carefully engineered.

They transmit time series of the sensed phenomenon to the central nodes where computations are performed and data are fused.

A sensor network is composed of a large number of sensor nodes, which are densely deployed either inside the phenomenon or very close to it. The position of sensor nodes need not be engineered or predetermined. This allows random deployment in inaccessible terrains or disaster relief operations. On the other hand, this also means that sensor network protocols and algorithms must possess self-organizing capabilities. Another unique feature of sensor networks is the cooperative effort of sensor nodes. Sensor nodes are fitted with an on-board processor. Instead of sending the raw data to the nodes responsible for the fusion, sensor nodes use their processing abilities to locally carry out simple computations and transmit only the required and partially processed data.

The above described features ensure a wide range of applications for sensor networks. Some of the application areas are health, military, and security.

Sensor networks can also be used to detect foreign chemical agents in the air and the water. They can help to identify the type, concentration, and location of pollutants. In essence, sensor networks will provide the end user with intelligence and a better understanding of the environment. We envision that, in future, wireless sensor networks will be an integral part of our lives, more so than the present-day personal computers.

Realization of these and other sensor network applications require wireless ad hoc networking techniques. Although many protocols and algorithms have been proposed for traditional wireless ad hoc networks, they are not well suited for the unique features and application requirements of sensor networks. To illustrate this point, the differences between sensor networks and ad hoc networks are outlined below:

- The number of sensor nodes in a sensor network can be several orders of magnitude higher than the nodes in an ad hoc network.

- Sensor nodes are densely deployed.

- Sensor nodes are prone to failures.

- The topology of a sensor network changes very frequently. 
- Sensor nodes mainly use broadcast communication paradigm whereas most ad hoc networks are based on point-to-point communications.

- Sensor nodes are limited in power, computational capacities, and memory.

- Sensor nodes may not have global identification (ID) because of the large amount of overhead and large number of sensors.

Since large number of sensor nodes are densely deployed, neighbor nodes may be very close to each other. Hence, multi-hop communication in sensor networks is expected to consume less power than the traditional single hop communication. Furthermore, the transmission power levels can be kept low, which is highly desired in covert operations.

Multi-hop communication can also effectively overcome some of the signal propagation effects experienced in long-distance wireless communication.

One of the most important constraints on sensor nodes is the low power consumption requirement. Sensor nodes carry limited, generally irreplaceable, power sources. Therefore, while traditional networks aim to achieve high quality of service (QoS) provisions, sensor network protocols must focus primarily on power conservation. They must have inbuilt trade-off mechanisms that give the end user the option of prolonging network lifetime at the cost of lower throughput or higher transmission delay.

Many researchers are currently engaged in developing schemes that fulfill these requirements. In this paper, we present a survey of protocols and algorithms proposed thus far for sensor networks. Our aim is to provide a better understanding of the current research issues in this field. We also attempt an investigation into pertaining design constraints and outline the use of certain tools to meet the design objectives.

\section{Related Work}

The selection of a routing protocol for a certain network depends on the information available at the network nodes and the communication overhead that can be tolerated. Table.1 presents taxonomy of existing routing approaches in this respect. In networks where each node is provided with full topology information, and which is stable over time, such as interconnection networks (e.g., hypercube and meshes) used for parallel computing, the shortest (possibly weighted) path algorithm can be applied to route a message from any source to any destination. Large networks with reasonably stable nodes over time, where autonomous nodes do not know the full network graph (e.g., the Internet), require a distributed routing approach. For this type of network, the Bellman-Ford protocol is mostly applicable. According to this protocol, nodes periodically exchange routing tables that contain the first hop toward "strategic" destinations and corresponding costs with neighbors in order to update their own tables. Each node checks whether the cost of routing via a given neighbor is smaller than that currently recorded in its routing table, and updates its next hop toward the destination if so.

\begin{tabular}{|l|l|l|}
\hline Centralized & Shortest (weighted) path & $\begin{array}{l}\text { Interconnection } \\
\text { networks }\end{array}$ \\
\hline Distributed & Bellman-Ford & Internet \\
\hline Localized- flooding & Route discovery & $\begin{array}{l}\text { Dynamic ad hoc } \\
\text { networks }\end{array}$ \\
\hline $\begin{array}{l}\text { Localized- path } \\
\text { based }\end{array}$ & $\begin{array}{l}\text { Greedy, Cost/progress } \\
\text { ratio }\end{array}$ & $\begin{array}{l}\text { Sensor } \\
\text { networks }\end{array}$ \\
\hline
\end{tabular}

Table 1. Communication overhead based taxonomy of routing protocols

In the same way, the centralized approach also calculates the cost to progress ratio by sending the information of each node to one centralized node and cost is measured against progress made towards the destination. In the localized method the routing is done by communicating the each node with its neighbor nodes. And the methodology of calculating the cost , progress made and packet transmission of a proposed method is also mentioned in this paper.

\section{Routing In Sensor Networks}

We first address the routing task when nodes are equipped with position information. Each node is assumed to know the position of itself, all its neighbors, and destination $D$. In addition, local knowledge at each node includes the cost of each of its links to neighboring nodes. The position of neighbors may be gained via 
periodic exchange of "hello" messages. In sensor networks the destination is normally a sink whose position is flooded to all sensors prior to their reporting.

\section{Cost to the Progress Ratio Routing Framework}

We now explain the general framework for our localized routing scheme. Suppose that each edge has a cost measure. The cost measure depends on the assumptions and metrics used, while progress measures the advance toward the destination.

In the next section we study specific cost measures such as hop count, power, reluctance, power reluctance, delay, and expected hop count. Figure 1 shows an example with costs listed. If global knowledge is available at each node, the optimal (shortest weighted) path from source $A$ to destination $B$ would be $A C D E F G M D$ (the total cost $2+3+1+5+8+24+6$ is minimal among total costs of all paths between $A$ and $B)$.However, we assume that each node has only local information. Thus, source $A$ has five choices to forward a packet to $B$, neighbors $C, D, E, I$, and $J$, and no knowledge of other nodes in the network except $B$. What is the best choice for $A$, based on such local information? We argue that the cost of the selected link should be evaluated against the progress made toward the destination. We measure the progress at the reduction in distance to destination of the current node to the distance of its neighbor. That is, ratios $2 /(|A B|-|\mathrm{AC}|), 24 /(|\mathrm{AB}|-$ $|\mathrm{AD}|), 38 /(|\mathrm{AB}|-|A E|), 18 /(|\mathrm{AB}|-|\mathrm{AI}|)$ and $10 /(|\mathrm{AB}|-|\mathrm{AJ}|)$ are compared, and the minimal one is selected. The rationale for the method is that it attempts to minimize the total cost by favoring neighbors closer to the destination (thus reducing the number of hops) and with smaller costs, with a suitable simple formula to choose a "winner "at each step. The cost to progress ratio methodology is applied for all the mentioned approaches.



Fig. 1. A sensor network with costs at each edge.

\section{Bellman Ford Asynchronous Sleep Wake Up Protocol}

The conventional Bellman Ford protocol uses significant communication overhead during route identification \& message transmission because it involves all the nodes in the alternate routes. In the proposed Bellman ford asynchronous sleep wake up protocol, the downstream node in the selected route is made to wake up by an alarm signal from upstream node, which remain active till required time and go to sleep, where as other nodes are in sleep mode. Thus, the proposed protocol significantly reduces energy consumption without incurring communication overhead and clock synchronization.

\section{ALGORITHM}

1) $i$ is a node, if $i=0 \&$ if $i$ is less than value then $i$ is incremented.

2) Set or create sleep_app.

i) Node transmission depends on the flag condition.

ii) If flag $==0$ then stop transmission $\&$ set the node condition==idle.

iii) $\quad$ Set flag $==1$ for transmission.

3) Create or set sleep_rreq_app new app.

i) Sleep_rreq will take sleep_app update init MAX received data node source destination.

ii) $\quad$ Set flag $==0$, if source $==$ data to be transmitted \& if $\operatorname{RREP(destination)}==$ received(source) then start data transmission, else RREQ updated, then transmit RREQ,initialize transmission of RREQ_MAX.

4) Create or set transmit data new app.

Transmit dropped RREQ, if source==data(received) $\quad$ \& if data(received)! $=$ RREQ then set flag==0, if flag $==0$ then

i) drop RREQ,otherwise transmit RREQ.

ii) Else if, destination $==$ data(received) \& node_id(RREQ) $==$ node_id(RREP) then initialize RREP.

5) Create or set sleep_rrep_app new app.

i) Sleep_rrep will have updated count, ack, init MAX received data node source. 
ii) If flag $==0$, then RREP \& ack \& data transmit, to make transmission flag 1 now, RREP, ack \& data can be transmitted.

iii) If node (received) !=source, then transmit it to the new node RREP, ack \& data else if set flag $1 \&$ if node $==0$ \& if count $=0$, transmit count \& stop RREP.

\section{Implementation}

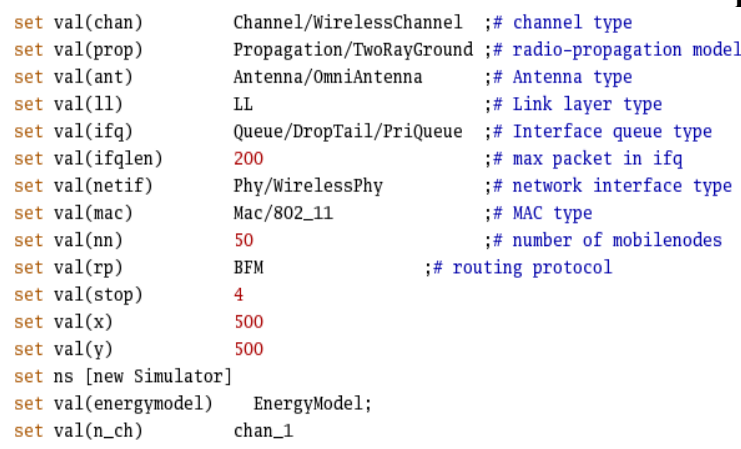

The implementation of proposed Bellman ford asynchronous sleep wake up protocol is done on Network Simulator 2(NS2) and the platform is Red Hat Linux. The code for the proposed algorithm developed at the backend of NS2 and simulation results obtained at the front end of NS2,the code is executed by the ns command. The output obtained in Network Animator (NAM) window and graphs on Xgraph window.

\section{Simulation Results}

To validate the performance of proposed Bellman Ford asynchronous sleep wake up protocol, we simulate the protocol and utilize a network with 50 nodes on Network Simulator 2(NS2).



Fig. 3. Comparison between Centralized, Localized, Distributive(Bellman ford) and proposed Bellman ford asynchronous sleep wake up protocol based on Avg Delay.

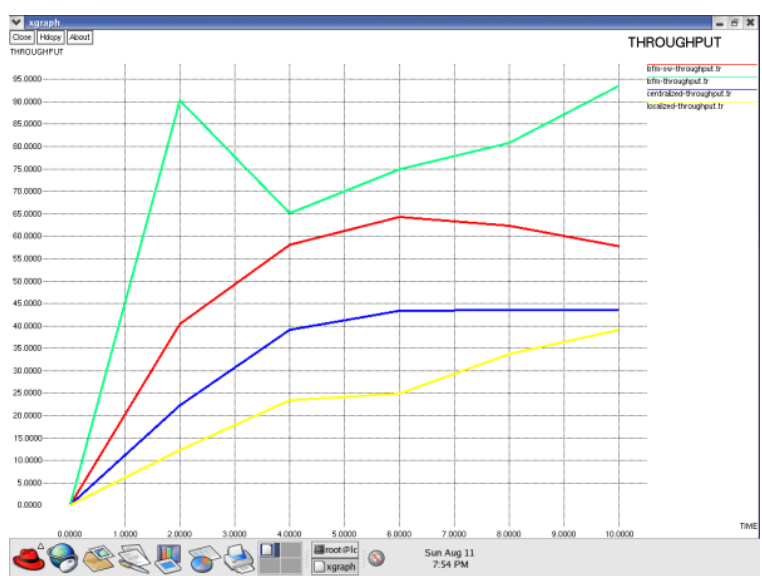

Fig. 4. Comparison between Centralized, Localized, Distributive(Bellman ford) and proposed Bellman ford asynchronous sleep wake up protocol based on Throughput. 


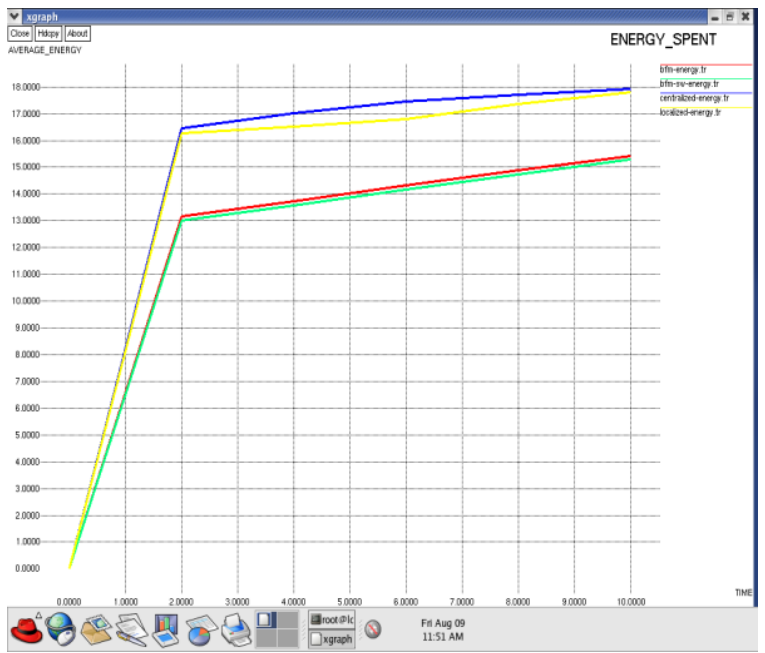

Fig. 5. Comparison of between centralized, localized, distributive and proposed bellman ford asynchronous sleep wake up protocol based on energy spent in packet transmission.

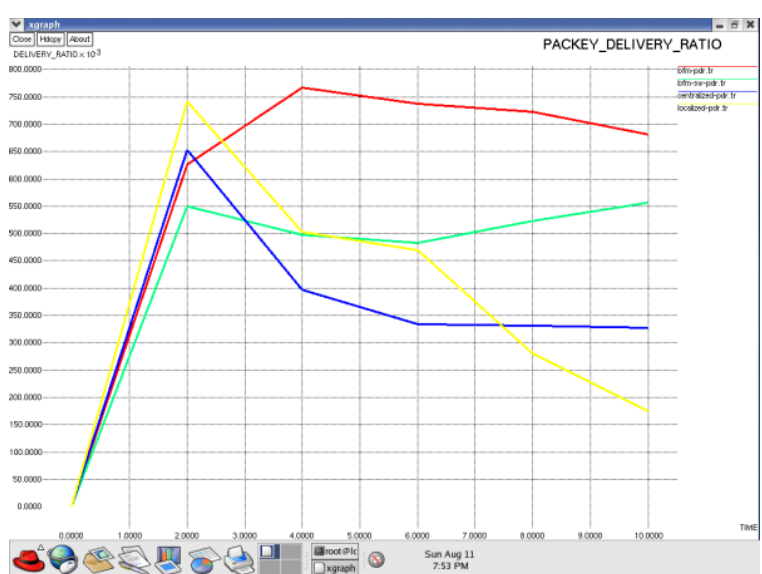

Fig. 6. Comparison between Centralized, Localized, Distributive(Bellman ford) and proposed Bellman ford asynchronous sleep wake up protocol based on Avg Delay

\section{Conclusion}

We, presented in this paper that, the three conventional network layer protocols and proposed Bellman ford asynchronous sleep wake up network layer protocol in wireless sensors networks and their simulated results on network simulator 2(NS2), after observing the graphs obtained on metrics like avg delay, energy spent, pdr, throughput, the energy spent in transmission of packets is reduced in the proposed method. The asynchronous sleep wake up protocols have more delays in transmission of packets. So, this delay may be reduced by more research in computation of routing.

\section{References}

[1] *Ivan Stojmenovic, University of Ottawa, Localized Network Layer Protocols In Wireless Sensor Networks Based on Optimizing Cost over progress Ratio,IEEE Networks 2006

[2] Low Power, Low Delay:Opportunistic Routing meets Duty Cycling-Olaf Landsiedel_ 1, Euhanna Ghadimi2, Simon Duquennoy3, Mikael Johansson2 olafl@chalmers.se, euhanna@kth.se, simonduq@sics.se, mikaeli@kth.se i)Chalmers University of Technology, Sweden ii)KTH Royal Institute of Technology, Sweden iii)Swedish Institute of Computer Science (SICS), Sweden

[3] C. Huang, F. Dai, and J. Wu, "On-Demand Location-Aided QoS Routing in Ad Hoc Networks," Proc. Int'l. Conf. Parallel Proc., Montreal, Canada, Aug. 2004.

[4] T. He et al., "A Spatiotemporal Communication Protocol for Wireless Sensor Networks," IEEE Int'l. Conf. Distrib. Comp. Sys., May 2003; to appear, IEEE Trans. Parallel and Distrib. Sys

[5] I. Stojmenovic, A. Nayak, and J. Kuruvila, "Design Guidelines for Routing Protocols in Ad Hoc and Sensor Networks with a Realistic Physical Layer," IEEE Commun. Mag., Ad Hoc and Sensor Networks Series, vol. 43, no. 3, Mar. 2005, pp. 101-06.

[6] I. Stojmenovic and X. Lin, "Power Aware Localized Routing In Ad Hoc Networks," IEEE Trans. Parallel and Distrib. Sys., vol. 12, no. 10, Oct. 2001, pp. 1023-32.

[7] M. R. Souryal, B. R. Vojcic, and R. L. Pickholtz, "Information Efficiency of Multihop Packet Radio Networks with ChannelAdaptive Routing," IEEE JSAC, vol. 23, no. 1, 2005, pp. 40-50.

[8] M. Mauve et al., "Position-Based Multicast Routing for Mobile Ad Hoc Networks," TR-03-004, Dept. Comp. Sci., Univ. of Mannheim, Mar. 2003; ACM Mobihoc Poster, 2003.

[9] P. M. Ruiz and I. Stojmenovic, "Cost-efficient Multicast Routing in Ad Hoc and Sensor Networks," to appear, Handbook on Approximation Algorithms and Metaheuristics, T. Gonzalez, Ed., Chapman \& Hall/CRC. 